\title{
Endoplasmic reticulum protein 29 (ERp29), a protein related to sperm maturation is involved in sperm-oocyte fusion in mouse
}

\author{
Xiaogian Ying ${ }^{\dagger}$, Yue Liư ${ }^{\dagger}$ Qiangsu Guo, Fei Qu, Wei Guo, Yemin Zhu, Zhide Ding*
}

\begin{abstract}
Background: Sperm-oocyte fusion is a critical step in fertilization, which requires a series of proteins from both spermatozoa and oocyte to mediate membrane adhesion and subsequent fusion. A rat spermatozoa membrane protein is endoplasmic reticulum protein 29 (ERp29), which significantly increases on the sperm surface as well as in the cytoplasm of epididymal epithelia from caput to cauda as the sperm undergo epididymal maturation. Moreover, ERp29 facilitates viral infection via mediating membrane penetration. We determined if in addition to promoting sperm maturation ERp29 may also play a role in facilitating gamete fusion during the fertilization process.

Methods: Laser scanning confocal microscopy (LSCM) and Western blot analysis were employed to probe for ERp29 protein in BALB/C mouse epididymal and acrosome-reacted spermatozoa. We prepared rabbit polyclonal antibodies against mouse recombinant ERp29 (rERp29) to characterize: 1) fertilization rate (FR); 2) fertilization index (FI); 3) sperm motility and 4) acrosome reaction (AR).

Results: Confocal microscopy indicated that ERp29 was partially localized at the sperm head of the epididymal caput as well as over the whole head and part of the principal piece of the tail region from the epididymal cauda. However, when the acrosome reacted, ERp29 remained in the equatorial and post-acrosomal regions of the sperm head, which is the initial site of sperm-oocyte membrane fusion. Such localization changes were confirmed based on the results of Western blot analysis. Furthermore, the antibodies against mouse rERp29 inhibited the spermatozoa from penetrating into the zona pellucida (ZP)-free oocytes. The functional blocking antibodies reduced both mouse sperm-oocyte FR and Fl at concentrations of 100 and 200 micro g/ml compared with preimmunized rabbit IgG or with anti-mouse recombinant bactericidal/permeability-increasing protein (BPI, a sperm surface protein unrelated to sperm-oocyte fusion) antibodies (100 micro $\mathrm{g} / \mathrm{ml}$ ), but they had no effect on sperm motility and AR.

Conclusion: This study demonstrates that ERp29 on mouse spermatozoa membrane changes during epididymal transit and AR. Accordingly, in mice this protein may be one of the important factors involved in sperm fertilization by facilitating sperm-oocyte membrane fusion.
\end{abstract}

\section{Background}

Sperm-egg plasma membrane interaction referred to as gamete fusion, is an extremely important step needed for mammalian fertilization [1]. In the past ten years, the mechanism of sperm-oocyte fusion has been

\footnotetext{
* Correspondence: zding@shsmu.edu.cn

† Contributed equally

Shanghai Key Laboratory for Reproductive Medicine, Department of Histology and Embryology, School of Medicine, Shanghai Jiao Tong University, Shanghai 200025, China
} and the ZP-free oocyte insemination test suggested that several sperm membrane proteins were involved in this process, e.g., a disintegrin and metalloproteinase gene family members (ADAM) [2-4], cysteine-rich secretory protein (CRISP) [5,6] and Izumo [7]. Although these proteins appear to play a role in sperm-oocyte fusion, it is evident that there are many other unidentified essential proteins participating in this process. 
Endoplasmic reticulum proteins (ERp), some of which are in the protein disulfide isomerase (PDI) family, play vital roles in protein secretion. In some cases, they are recognized primarily as catalysts of disulphide bond formation and chaperones of protein folding [8]. Recent reports showed that a $57 \mathrm{kDa}$ ERp, ERp57, participate in sperm-oocyte fusion and is intimately associated with providing human sperm fertilization capability $[9,10]$. However, only a few of the described ERps have been identified in mammal spermatozoa or reported to be involved in spermatozoa fertility.

In our previous study, we first reported ERp29 in rat spermatozoa and confirmed that ERp29 was increased significantly on the sperm surface as well as in the cytoplasm of epididymal epithelia from caput to cauda during sperm epididymal maturation [11]. Furthermore, ERp29 could facilitate polyomavirus to infect a host cell via triggering a conformational change in virus and stimulating membrane binding [12]. We undertook the present study to verify ERp29 expression on BALB/c mouse sperm during epididymal transit and acrosome reaction, and further investigated the ERp29's functional role in sperm-oocyte fusion.

\section{Methods}

\section{Chemicals and animals}

Unless otherwise stated, all reagents were purchased from Sigma-Aldrich Corporation (St. Louis, MO, USA). Animal experiments were conducted according to the International Guiding Principles for Biomedical Research Involving Animals, as promulgated by the Society for the Study of Reproduction. White New Zealand rabbits (male: $\sim 6$ months old, body weight $\sim 2.5 \mathrm{~kg}$ ) and BALB/c mice (male: aged 9-11 weeks, body weight 22-24 g; female: aged 7-9 weeks, body weight 18-20 g) were purchased from Shanghai SLAC Laboratory Animal Corporation (Jiu-Ting, Shanghai, China) and accommodated in the animal facility for at least for 1 week prior to experimentation.

\section{Preparation of epididymal spermatozoa, sperm protein and membrane protein}

Isolation of mouse epididymal caput and caudal spermatozoa, and protein extraction were performed as described [11]. Sperm membrane protein was isolated with the ReadyPrep Protein Extraction Kit (Pierce, Rockford, IL, USA). Protein concentration was determined by $\mathrm{BCA}^{\mathrm{ma}}$ Protein Assay Kit (Pierce), using bovine serum albumin (BSA) as a protein standard.

\section{Western blot analysis}

Protein samples $(20 \mu \mathrm{g}$ per well) were separated under denaturing conditions using SDS-PAGE, and then transferred to polyvinylidene difluoride (PVDF) membranes
(GE Healthcare, Waukesha, WI, USA) using a semi-dry transfer apparatus (Bio-Rad, Hercules, CA, USA). Membranes were blocked for $1 \mathrm{~h}$ at room temperature with Tris-buffered saline (TBS) containing $0.1 \%$ Tween-20 and $5 \%$ BSA. Immunoblotting was performed with rabbit polyclonal ERp29 antibody (Abcam, Cambridge, MA, USA) at a 1:1000 dilution, followed by incubation with secondary antibody conjugated to HRP (Abgent, San Diego, CA, USA) at a 1:5000 dilution. The blotting signals were detected by enhanced chemiluminescence (ECL) (ECL Plus, GE Healthcare), following the manufacturers' protocol. In the meantime, $\beta$-actin served as the internal control. Western blot analysis was repeated three times and then the results were scanned by Calibrated Densitometer (Bio-Rad GS-800). Finally, the averages were calculated with Quantity One (Version 4.6.1) software.

\section{ERp29 immunofluorescent staining}

Mouse caput and caudal spermatozoa were isolated and immediately smeared onto individual slides. ERp29 immunofluorescent staining was performed as described [11]. Slides were blocked with 5\% BSA and incubated with rabbit anti-mouse ERp29 antibodies (Abcam) or normal rabbit IgG (Upstate, Temecula, CA, USA) (1:500 dilution each). Goat anti-rabbit antibody linked with fluorescein isothiocyanate (FITC) (Rockland, Gilbertsville, PA, USA) was used as the secondary antibody at 1:300 dilution. Finally, the fluorescent-stained sperm samples were viewed under LSCM (Carl Zeiss LSM-510, Jena, Germany) and the graphs were processed with its accompanying AIM (Release Version 4.0 SP2) software. Indirect immunofluorescence analysis was repeated at least three times.

\section{Prokaryotic expression and purification of recombinant ERp29}

Total RNA was extracted from mouse liver with RNA Easy (Qiagen, Valencia, CA, USA), according to the manufacturer's protocol. Then, RNA was transformed into cDNA with RT-PCR. A segment of ERp29 was amplified with the forward primer 5'-GCGGATCCTTC TACAAGGTCATTCCC-3'(containing BamH I site, bolded) and the reverse primer 5'-GCAAGCTTTAGCC CACTTCTTCTCTG-3'(containing Hind III site, bolded). Prokaryotic expression and purification of rERp29 were performed as previously described [13]. The new recombinant prokaryotic expression vector was named pET-28a(+)/ERp29, and propagated in E. coli BL21 (DE3) host cells cultured with $0.8 \mathrm{mM}$ isopropylb-Dthiogalactopyranoside (IPTG) for 12 hours at $37^{\circ} \mathrm{C}$ with gentle shaking. The purified protein was freezedried in a Heto Freeze Dryer (Thermo Electron Corporation, Waltham, MA, USA), and its identity was confirmed with 15\% SDS-PAGE analysis and Western blot using anti-His monoclonal antibody (Abgent). 
Production of polyclonal rabbit anti-rERp29 antibodies Production of polyclonal rabbit anti-mouse rERp29 antibodies was performed as described [13]. The titer of the antiserum was measured using an indirect ELISA at 450 $\mathrm{nm}$ in conjunction with an Anthos Zenyth 1100 multimode detector (Anthos Labtec Instruments $\mathrm{GmbH}$, Wals, Austria) with a 5-s pre-read shaker. Finally, the immunized rabbit IgG (including anti-rERp29 IgG) was purified through immunoaffinity chromatography from crude rabbit sera, using the ImmunoPure (G) IgG Purification kit (Pierce). In the meantime, normal rabbit IgG was purified from pre-immunized rabbit sera.

\section{Gametes preparation}

Female BALB/c mice were superovulated by abdominal injection of $10 \mathrm{IU}$ of pregnant mare's serum gonadotropin (PMSG; PROSPEC, Rehovot, Israel), followed 46-48 hours later by $10 \mathrm{IU}$ of human chorionic gonadotropin (hCG; Li Zhu drug plant, Zhuhai, China). Cumulus enclosed-egg complexes were collected from the oviducts 15-16 hours after post-hCG injection, and treated with $0.1 \%$ hyaluronidase in Medium 16 (M16) to disperse the cumulus cells. The ZP was removed with $0.6 \%$ proteinase $K$, and then the eggs were incubated with M16 containing 3\% BSA at $37^{\circ} \mathrm{C}, 5 \% \mathrm{CO}_{2}$ for $30-60$ minutes.

Mature spermatozoa were collected from epididymal cauda of BALB/c male mice by rough cutting in $1.5 \mathrm{ml}$ of M16 containing 3\% BSA; Motile spermatozoa were allowed to swim out for 10-15 minutes, and then the tissue was removed from the medium. One part of spermatozoa were cultured for 2 hours in M16 (containing $3 \mathrm{mg} / \mathrm{ml} \mathrm{BSA}$ and $5 \mu \mathrm{mol} / 1$ ionophore A23187) at $37^{\circ} \mathrm{C}$ and $5 \% \mathrm{CO}_{2}$, then used for localization of ERp29 in acrosome-reacted spermatozoa. The resting spermatozoa were cultured for 90 minutes in M16 containing $3 \mathrm{mg} /$ $\mathrm{ml} \mathrm{BSA}$ at $37^{\circ} \mathrm{C}, 5 \% \mathrm{CO}_{2}$, allowing them to capacitate and undergo spontaneous AR. Following that, spermatozoa (adjusted to $10^{7}$ sperm $/ \mathrm{ml}$ ) were incubated in the presence or absence of prepared and purified rabbit anti-mouse rERp29 antibodies (without sodium azide) or pre-immunized rabbit IgG (normal rabbit IgG) at different concentrations $(20,50,100,200 \mu \mathrm{g} / \mathrm{ml})$, or rabbit anti-mouse rBPI antibodies $(100 \mu \mathrm{g} / \mathrm{ml}$, the antibodies were a generous gift from Dr Zhongping Zhou form at the Shanghai Key Laboratory for Reproductive Medicine, Shanghai, China), or washing buffer (control) for 40 minutes, and then diluted (1:40) with M16 containing $3 \%$ BSA for use in the sperm-oocyte fusion assay and the final sperm concentration was $2.5 \times 10^{5} \mathrm{sperm} / \mathrm{ml}$.

\section{Sperm-oocyte fusion assay}

For every sperm-oocyte fusion assay, all the oocytes and spermatozoa used in an experiment were combined from several female and male mice and then divided into each group randomly. ZP-free oocytes were added directly to the sperm suspensions prepared as described above, and the gametes were co-incubated for 3 hours at $37^{\circ} \mathrm{C}, 5 \% \mathrm{CO}_{2}$. Then, loosely bound spermatozoa were removed from the oocytes by gentle pipetting. After that, the oocytes were fixed with $4 \%$ paraformaldehyde for 15 minutes, and then treated with Hoechst 33342 for $10 \mathrm{~min}$ to stain the chromatin. Finally, images were collected using the LSCM and results were evaluated as the percentage of oocytes penetrated by sperm (FR) and the average number of penetrated sperm per oocyte (FI). Each assay was repeated at least three times at each concentration of IgG used in every experiment.

\section{Assessment of sperm motility and acrosome reaction}

Spermatozoa from epididymal cauda were cultured for 90 minutes in M16 containing $3 \mathrm{mg} / \mathrm{ml} \mathrm{BSA}$ at $37^{\circ} \mathrm{C}$ and $5 \%$ $\mathrm{CO}_{2}$, and then incubated either with prepared anti-mouse rERp29 antibodies or with normal rabbit IgG at the concentrations of $20,50,100,200 \mu \mathrm{g} / \mathrm{ml}$ for 40 minutes. After sperm/antibody incubation, sperm motility (including movement parameters: velocity average path (VAP), velocity curvilinear (VCL) and velocity straight line (VSL)) was assessed using computer-assisted semen analysis (CASA; Hamilton-Thorn Research, Beverly, MA, USA) with parameters optimized for detection of mouse sperm.

AR assays were conducted essentially as described [14]. Sperm suspensions were fixed with $4 \%$ paraformaldehyde solution ( $\mathrm{pH}$ 7.4) for 10 minutes, and then centrifuged and washed twice with $100 \mathrm{mM}$ ammonium acetate ( $\mathrm{pH}$ 9.0). The final sperm pellet was resuspended in $100 \mathrm{mM}$ ammonium acetate, and $50 \mu \mathrm{l}$ of the sperm suspension was smeared on a glass slide and incubated with Coomassie Blue (0.22\% Coomassie Blue G-250, $50 \%$ methanol, $10 \%$ glacial acetic acid, $40 \%$ water) for 2 minutes. Finally, the percentage of acrosome-reacted cells was calculated by evaluating at least 200 spermatozoa and every experiment was repeated three times.

\section{Statistical analysis}

Data are presented as mean \pm SEM and analyzed using the SAS 8.2 statistical software. Fertilization rate was analyzed by chi-square test. Student-Newman-Kuel's test was performed to assess the significance of individual variations among the treated groups. Differences were considered statistically significantly different when $P<0.05$.

\section{Results}

Identification and localization of ERp29 on spermatozoa ERp29 was present in mouse spermatozoa from both epididymal caput and cauda. As indirect immunofluorescence showed, ERp29 was mostly localized on part of the spermatozoa head from the epididymal caput, whereas it was also localized on the whole head and in 
the tail region (principal piece) of spermatozoa from the cauda. Moreover, ERp29 was still present in mouse acrosome-reacted spermatozoa, mostly on the equatorial and post-acrosomal regions of sperm head, which are generally considered as the initial sites of sperm-egg fusion (Fig 1).

The results of Western blot analysis showed that the spot identified as ERp29, was significantly up-regulated in the sperm from caudal epididymis and then downregulated in acrosome reacted sperm. Gel protein loading equivalence is documented by invariant levels of $\beta$ actin expression (Figs 2A and 2B). The ERp29 content in whole sperm protein as well as membrane protein revealed the same changes as found using indirect immunofluorescence analysis. This agreement confirmed that the ERp29 protein content was higher in the caudal sperm than in the caput or in acrosome-reacted sperm, especially on the sperm membrane (Figs 2C and 2D).

\section{Prokaryotic expression and purification of rERp29}

To overexpress the rERp29 protein and raise antibodies, a protein fragment of good antigenicity was selected by DNAssist. A 463 bp fragment was amplified from Mus musculus cDNA by PCR and separated on a $1 \%$ agarose gel (Fig 3A). The target fragment of ERp29 was then cloned into the prokaryotic expression vector, which was named pET-28a(+)/ERp29 (Fig 3B). This construct is predicted to encode a 206 amino acid-containing recombinant protein with molecular weight of $\sim 23 \mathrm{kDa}$. It was successfully overexpressed by inducing it with IPTG; this $23 \mathrm{kDa}$ protein was absent in non-induced cells, shown on $15 \%$ SDS-PAGE gel (Fig 4A). The recombinant protein was purified and then confirmed by SDS-PAGE analysis (Fig 4A) and Western blot using anti-His monoclonal antibody (MAB) (Fig 5A).

\section{Production of polyclonal anti- rERp29 antibodies in rabbits}

Polyclonal antibodies against the rERp29 protein were generated by immunization of white New Zealand rabbits. At day 35, rabbits were killed and sera were collected. Results from the indirect ELISA indicated that the titers of rabbit anti-rERp29 sera were fairly high compared with that of pre-immune serum (control), reaching 1:128000 (Fig 4B). Anti-rERp29 IgG (without sodium azide) was then purified from crude rabbit sera, confirmed by Western blot (Fig 5B) and stored at $4^{\circ} \mathrm{C}$ for functional analysis in vitro.

\section{Effects of ERp29 on sperm-oocyte fusion studied by rERp29 antibodies}

To assess the possible role of ERp29 in mouse spermoocyte fusion during fertilization, we tested whether anti-rERp29 antibodies could inhibit mouse sperm from

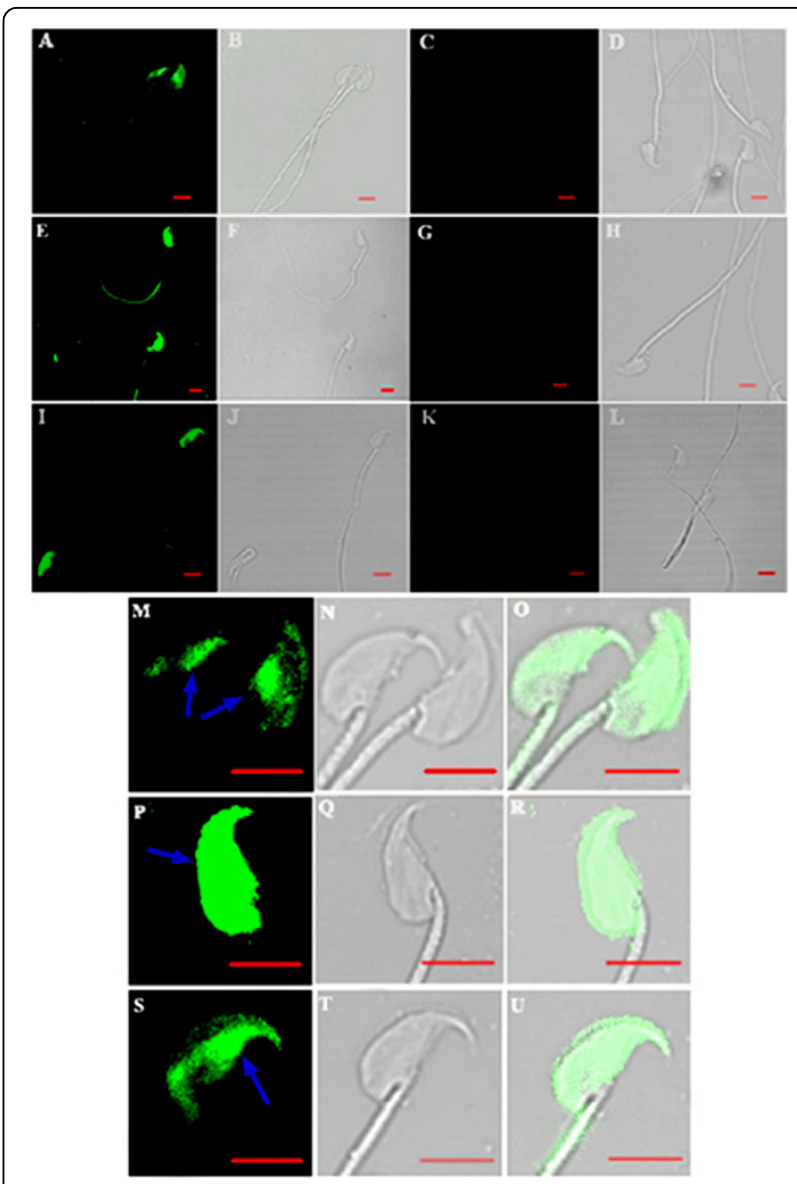

Figure 1 Localization of ERp29 on BALB/c mouse spermatozoa by indirect immunofluorescence analysis. (A), (E), (I) The localization of ERp29 on spermatozoa from epididymal caput, caudal regions and on acrosome-reacted spermatozoa. $(B),(F),(J)$ Differential interference contrast (DIC) images corresponding to (A), $(\mathrm{E}),(\mathrm{I}) .(\mathrm{C}),(\mathrm{G}),(\mathrm{K})$ Caput, caudal and acrosome-reacted spermatozoa incubated with normal rabbit lgG as negative controls. $(D),(H),(L)$ DIC images corresponding to $(C),(G),(K)$. (M),(N) Caput sperm head in $(A),(B)$ were magnified to show the precise localization. $(P),(Q)$ Caudal sperm head in $(E),(F)$ were magnified to show the precise localization. (S),(T) Acrosome-reacted sperm head in $(I),(J)$ were magnified to show the precise localization. $(\mathrm{O}),(\mathrm{R}),(\mathrm{U})$ The combined image of $(M)$ and $(N),(P)$ and $(Q),(S)$ and $(T)$, respectively. The arrows indicated the exact localization of ERp29 in the spermatozoa on different conditions. Calibration bar $=5 \mu \mathrm{m}$.

penetrating ZP-free oocytes. Thus, anti-mouse rERp29 antibodies (without sodium azide) generated from rERp29 immunized rabbits were used in these tests. Both the FR and FI were used to evaluate sperm ability to fertilize oocytes. Lower percentages of oocytes penetration were detected in anti-rERp29 antibodies treated sperm at the concentration of $100,200 \mu \mathrm{g} / \mathrm{ml}(0.71 \pm$ $0.08,0.56 \pm 0.09$, respectively) compared with normal rabbit IgG (from pre-immunized sera) treated sperm $(0.85 \pm 0.04,0.82 \pm 0.08$, respectively), or with anti-rBPI antibodies treated sperm $(100 \mu \mathrm{g} / \mathrm{ml}, 0.87 \pm 0.01)$, or 

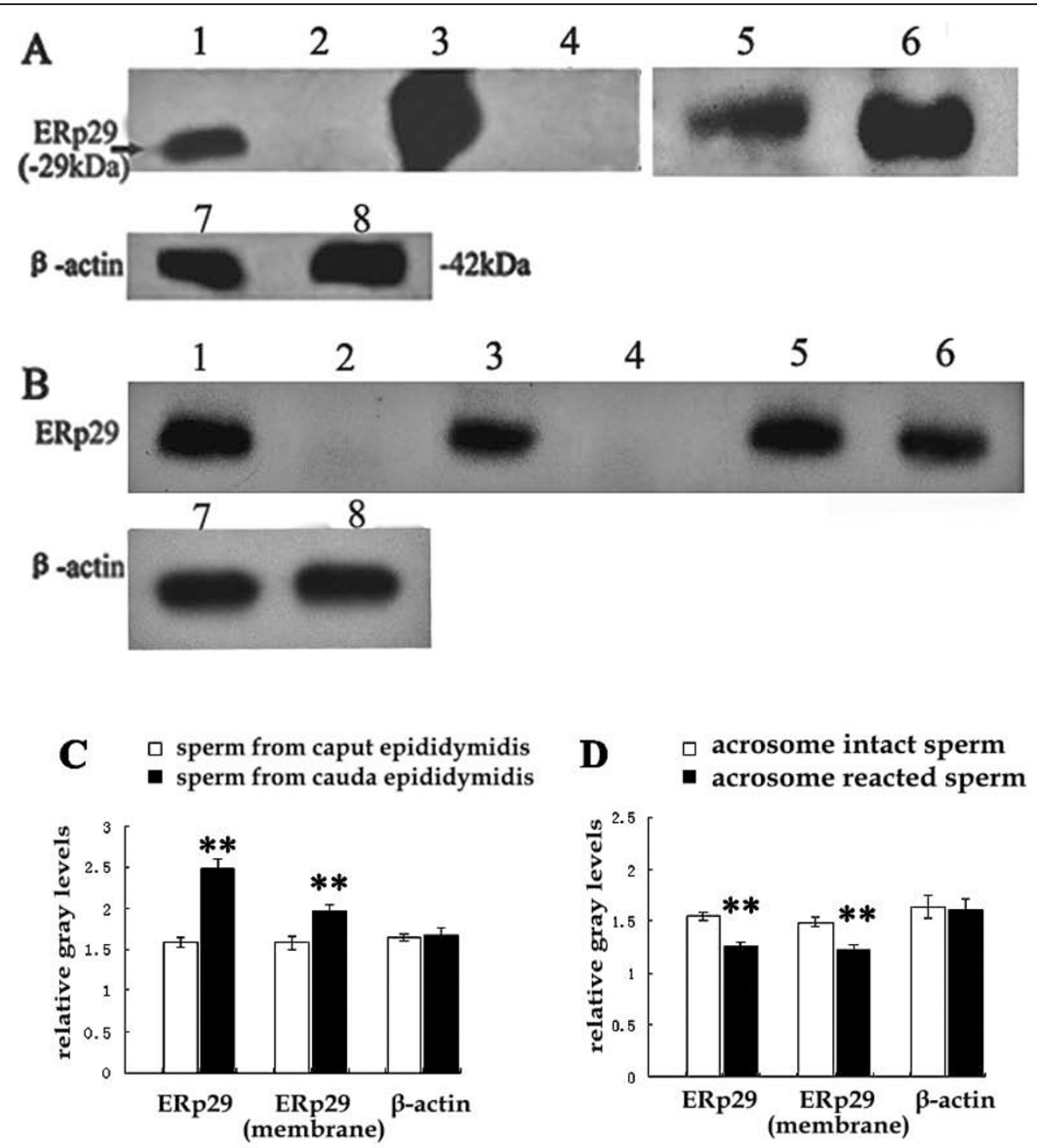

Figure 2 Western blot analysis of ERp29 in BALB/c mouse epididymal and acrosome-reacted sperm. (A) Lane 1, ERp29 in the whole protein from caput sperm; Lane 3, ERp29 in the whole protein from caudal sperm; Lane 5, ERp29 in the membrane protein from caput sperm; Lane 6, ERp29 in the membrane protein from caudal sperm; Lane 2 and 4, Normal rabbit lgG as negative control; Lanes 7 and 8, $\beta$-actin in the whole protein from caput and caudal sperm served as loading control. (B) Lane 1, ERp29 in the whole protein from acrosome intact sperm; Lane 3, ERp29 in the whole protein from acrosome-reacted sperm; Lane 5, ERp29 in the membrane protein from acrosome intact sperm; Lane 6, ERp29 in the membrane protein from acrosome-reacted sperm; Lane 2 and 4, Normal rabbit lgG as negative control; Lane 7 and 8, $\beta$-actin in the whole protein from acrosome intact and reacted sperm served as the loading control. (C) Average gray scale levels of the corresponding protein blot in (A) repeated three times. (D) Average gray scale levels of the corresponding protein blot in (B) repeated three times $(* * P<0.01)$.

with control sperm $(0.88 \pm 0.02)$ (Fig $6 \mathrm{~A},{ }^{*} P<0.05$, *** $P$ $<0.01$; number of oocytes used in the experiments: Control $=81$; Anti-rBPI $=46$; Normal $=72$, 75; AntirERp29 = 76, 80; respectively). Similarly, sperm fertilization index values were also significantly lower in antirERp29 antibodies treated sperm at concentrations of $100,200 \mu \mathrm{g} / \mathrm{ml}(1.79 \pm 0.30,1.16 \pm 0.22$, respectively $)$ compared with normal rabbit IgG treated sperm (4.13 \pm $0.35,3.91 \pm 0.37$, respectively), or with anti-rBPI antibodies treated sperm $(100 \mu \mathrm{g} / \mathrm{ml}, 4.56 \pm 0.25)$, or with control sperm $(5.14 \pm 0.51)$ (Fig $6 \mathrm{~B}, * * P<0.01)$. In contrast to sperm, the anti-ERp29 antibodies had no significant effects on sperm-oocyte fusion when oocytes were pre-treated with the antibodies at a concentration of $100 \mu \mathrm{g} / \mathrm{ml}(P=0.12, P=0.34$, for FR and FI respectively).

\section{Effects of ERp29 on sperm motility and AR studied by rERp29 antibodies}

To assess the possible role of ERp29 in mouse sperm motility and AR during fertilization, we tested whether anti-rERp29 antibodies could inhibit or facilitate sperm motility and AR. After sperm/antibody incubation at different concentrations, sperm motility (including 


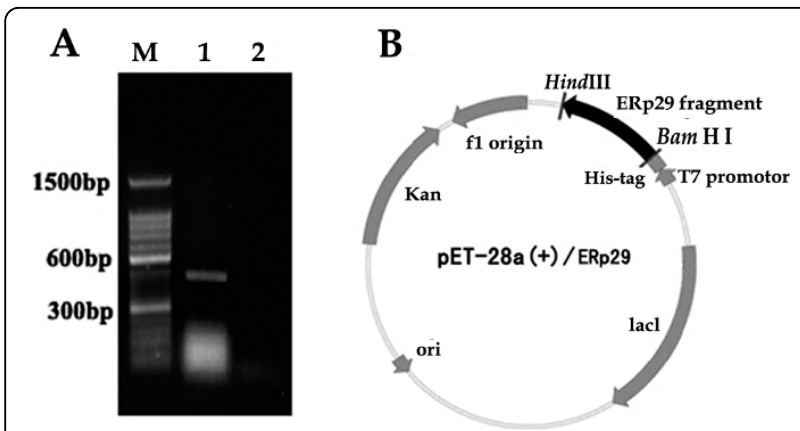

Figure 3 Cloning the target fragment of ERp29. (A) RT-PCR products of ERp29 fragment on 1\% agarose gel. Lane M, marker; lane 1, ERp29 fragment; lane 2, negative control (PCR without CDNA). (B) Schematic of the recombinant plasmid pET-28a(+)/ERp29.

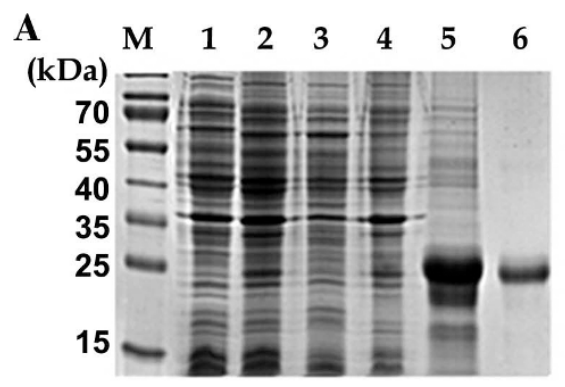

B

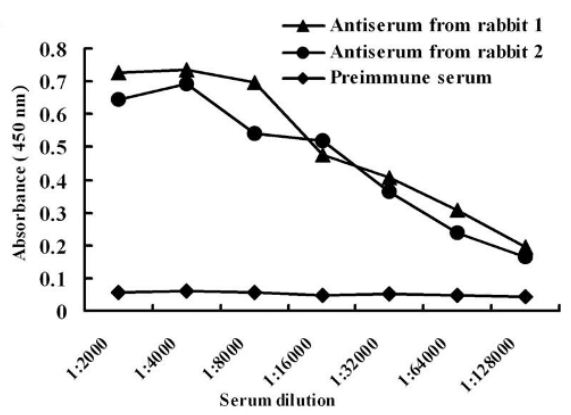

Figure 4 Prokaryotic expression and purification of rERp29, and production of polyclonal anti-rERp29 antibodies in rabbits. (A) Expression and purification of rERp29 fragment in E. coli BL21 (DE3) host cells. Different fractions of E. coli were separated on a Coomassie-blue-stained 15\% SDS-PAGE gel. Lane M, protein molecular weight markers; lane 1, total cell lysates carrying pET-28a (+)/ERp29 fragment before IPTG induction; lane 2, total cellular protein after IPTG induction; lane 3, supernatant of cell lysate after ultrasonic treatment; lane 4, pellet of cell lysate after ultrasonic treatment; lane 5, protein purified based on its His6-tag by affinity chromatography employing a $\mathrm{Ni}^{2+}$-NTA His-binding resin; lane 6 , protein eluted in PBS by cutting certain protein bands from the gel after preparative electrophoresis. (B) Values of ELISA absorbance at $450 \mathrm{~nm}$ using rERp29 protein immunized rabbit sera or pre-immune serum (control).

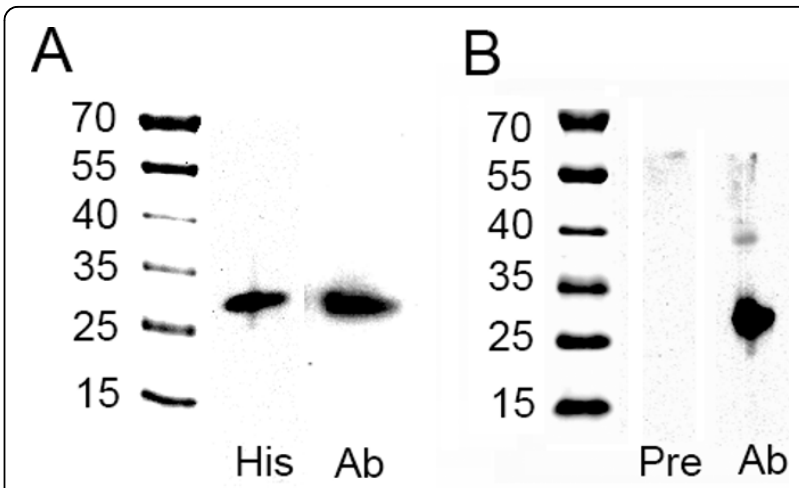

Figure 5 The purified rERp29 protein was analyzed by Western blot. (A) The purified rERp29 protein $(\sim 23 \mathrm{kDa})$ was confirmed by immunoblotting, using anti-His monoclonal antibody (1:1000 dilution) and rabbit anti-rERp29 IgG purified from sera (1:1000 dilution). (B) ERp29 ( $29 \mathrm{kDa}$ ) was distinctly detected in BALB/C mouse sperm proteins using rabbit anti-rERp29 IgG (1:1000) but not with IgG from pre-immunized sera. His, anti-His monoclonal antibody; Ab, rabbit anti-rERp29 IgG purified from sera; Pre, IgG from pre-immunized sera.

movement parameters: VAP, VCL and VSL) was assessed using CASA. Sperm acrosome status was evaluated by Coomassie blue G250 stain. The results show that there are no significant differences between them. Both sperm motility and AR were unchanged in treated sperm at all anti-rERp29 antibody concentrations relative to those found in either normal IgG treated sperm, or blank control ( $\mathrm{P}>0.05$ ) (Figs $7 \mathrm{~A}$ and $7 \mathrm{~B}$ ). These results demonstrate that anti-rERp29 antibodies can significantly inhibit mouse sperm from penetrating the ZPfree oocytes. Such suppression was not attributable to changes in either the sperm motility or acrosome status.

\section{Discussion}

ERp29 was first reported during proteomic investigations of mammalian tissues [15-17]. It is expressed ubiquitously in mammalian tissues [18-20] and the homologous proteins have been identified in organisms as primitive as the fruit fly [21]. As previously reported, ERp29 was widely thought to be an assistant for protein folding and has a probable function as a PDI like molecular chaperone. It may have a role in the production of endomembrane and secretory proteins, and also can bind to other ERps [19,20].

In our previous study, we found that ERp29 was apparently up-regulated as rat sperm underwent epididymal maturation and expressed mainly on caudal sperm [11]. We found in the current study that these changes also occur in BALB/c mouse. Our results indicate that in epididymal caput sperm, ERp29 was localized on the anterior region of head, whereas it was instead localized on the whole head and tail region 

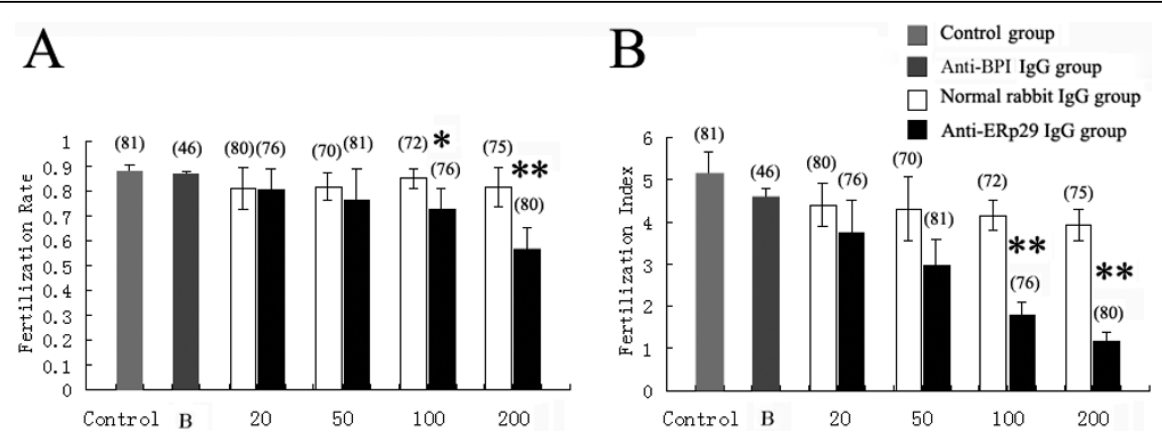

Figure 6 Effect of anti-rERp29 antibodies on sperm penetration assay. (A) FR at antibodies concentrations of 20, 50, 100, 200 $\mu \mathrm{g} / \mathrm{ml}$. (B) Fl at the antibodies concentration of 20,50,100,200 $\mu \mathrm{g} / \mathrm{ml}$ (B was anti-rBPl at the antibodies concentration of $100 \mu \mathrm{g} / \mathrm{ml}$; Data were presented as mean \pm SEM. $\left.{ }^{*} P<0.05,{ }^{*} P<0.01\right)$. For all statistical figures, total number of oocytes used in each group was marked above the column.

(middle pieces) in sperm from cauda. Besides, ERp29 was still present in the equatorial and post-acrosomal regions of the sperm head after acrosome reacted, which are generally considered as the initial sites of sperm-egg fusion. This distribution of ERp29 suggests that it may be an important factor for mature sperm function, especially for fertilization processes, such as capacitation, AR and fusion with oocytes.

It is noteworthy recent studies report that ERp29 can facilitate virus membrane penetration or fusion into

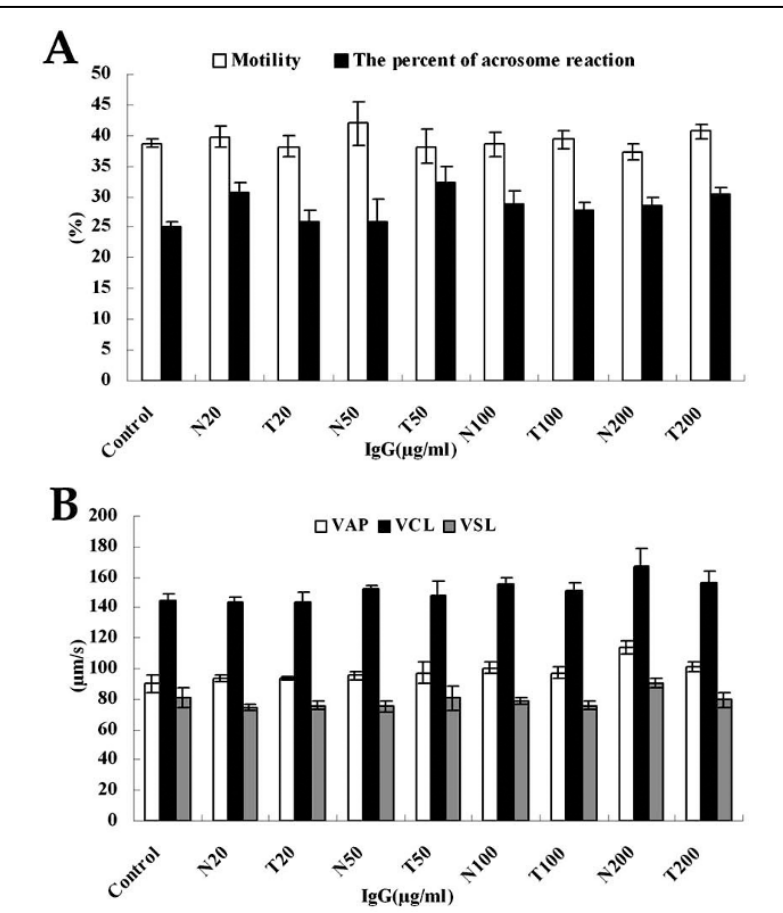

Figure 7 Effect of anti-ERp29 antibodies on sperm motility and AR assay. (A) Assessment of sperm motility and AR by different lgG treatments. (B) Assessment of sperm VAP, VCL and VSL by different IgG treatments (N: normal rabbit lgG; T: anti-rERp29 IgG; Values reported were mean $\pm \mathrm{SEM}, P>0.05)$. host cells $[12,22]$. Thus, we presumed that the expression pattern of ERp29 on mouse acrosome-reacted spermatozoa may also have a potential role in sperm fertilization, especially in sperm-oocyte membrane fusion. This presumption was tested by determining whether or not ERp29 present in the mouse spermatozoa membrane participates in sperm-oocyte fusion. Thus, we cloned the gene encoding mouse ERp29 and generated the relevant polyclonal antibodies. In our sperm penetration assay, we found that anti-mouse rERp29 antibodies could significantly reduce both mouse sperm-oocyte FR and FI at antibodies concentration of 100 and $200 \mu \mathrm{g} / \mathrm{ml}$, but they had no effects on sperm motility and AR. In contrast to sperm, the antiERp29 antibodies had no significant effects on spermoocyte fusion when the oocytes were instead pre-treated with these antibodies. Furthermore, in order to remove any concern about nonspecific effects from anti-ERp29 antibodies, we employed anti-mouse rBPI antibodies as one kind of antibody control. BPI protein is one of the membrane proteins from mouse sperm head, which is not involved in sperm-oocyte membrane fusion [23]. The FR and FI results revealed that there is no significant difference between the preimmune IgG group (normal IgG) and antibodies against rBPI at an antibody concentration of $100 \mu \mathrm{g} / \mathrm{ml}$, but a significant difference was observed between the anti-rBPI and anti-rERp29 groups. This strongly indicates that ERp29 may be involved in sperm fertilization by facilitating spermoocyte membrane fusion.

We addressed the question about how ERp29 affects sperm-oocyte membrane interaction. Until now there have no clear understanding of the molecular mechanisms underlying this phenomenon. Our results suggest that ERp29 on acrosome-reacted sperm membrane may play the same role as the PDI during sperm-oocyte fusion. Although PDIs have long been recognized as endoplasmic reticulum-resident proteins, recent studies have also found thiol isomerases on the surface of cells 
[24]. Gamete fusion may require a sperm surface-associated PDI, which can trigger a protein re-folding step leading to sperm-egg fusion. This mechanism relies on thiol-disulfide exchange, which may act in gamete fusion to produce conformational changes in fusion-active proteins [9]. PDI family members are characterized by the presence of a thioredoxin domain in their structures. Within this domain is the signature $\mathrm{CxxC}$ motif that allows the protein to cycle between a reduced and an oxidized state. In some cases, one of the Cys residues can be replaced by a different residue (e.g., CxxA). ERp29 has already been reported to be a member of PDI family [24] and contains a PDI-like structure: mature ERp29 (25.6 kDa, Mr 29,000 on SDS-PAGE) has an $\mathrm{N}$-terminal domain homologous to the thioredoxinlike domains in PDI, and a C-terminal domain with similarities to the P5 subfamily of PDI [25-29]. Due to the fact that ERp29 contains only a single Cys residue in its entire sequence (Cys157), it remains either in the reduced state or forms a mixed disulfide bond with another endoplasmic reticulum factor. Thus, ERp29's reduced and mixed-disulfide bonded states may exhibit different conformations, enabling it to bind to substrates with different affinities. Alternatively, noncovalent interactions with other ER factors could change ERp29's conformation to drive the substrate binding and release cycle [12].

Besides, the mechanism of sperm-egg fusion regulated by ERp29 may also be presumed from the results of a study describing polyomavirus entrance into the endoplasmic reticulum of a host cell [22]. Down-regulation of PDI in cells is recently observed to inhibit polyomavirus infection and ERp29 was shown to trigger a conformational change in polyomavirus [30]. In this regard, ERp29 can alter the conformation of polyomavirus' coat protein VP1, internal protein VP2 and then stimulate polyomavirus to bind and perforate ER membrane $[12,22]$. The data suggest that ERp29 renders polyomavirus virions hydrophobic, allowing the entire viral particle to bind to the surface of the endoplasmic reticulum membrane; this step may prepare the virus for penetration across the lipid bilayer. In the meantime, dimerization of ERp29, mediated by N-terminal domain, serves as a general mechanism to regulate its endoplasmic reticulum activities [31]. Moreover, both ERp29 N-terminal and $\mathrm{C}$-terminal domains are essential for inducing the local unfolding of polyomavirus to initiate the endoplasmic reticulum membrane penetration process [22,31,32]. Although the above explanations are all tenable based on our findings, there are additional questions that need to be addressed regarding the involvement of ERp29 in sperm-oocyte fusion. e.g., what is the relationship between Izumo and ERp29? Izumo is a critical factor involved in sperm-ooctye fusion localized on both mouse and human spermatozoa and detected exclusively in testis and sperm [7]. ERp29 and Izumo have poor sequence homology (only 33\% identity in a 36 amino acids fragment, analyzed by protein-protein BLAST at $\mathrm{NCBI}$ ). It remains unclear why both of them have effects on sperm-oocyte fusion, but have none on sperm AR.

\section{Conclusion}

Our current studies initially demonstrated that ERp29 is present on mouse sperm membrane and is apparently up-regulated as the sperm undergoes epididymal maturation. It still remains in the equatorial and postacrosomal regions of sperm head after AR. Anti-mouse rERp29 antibodies significantly reduced both mouse sperm-oocyte FR and FI, but they had no effect on sperm motility and AR. These results indicate that ERp29 has a potential function in mammalian fertilization and may be one of the novel factors in spermoocyte fusion.

\section{Acknowledgements}

The authors thank Dr Zhengmei Lv, Dr Zhongping Zhou, Ms Ruyao Wang, Ms Meige Lu, Ms Yanqin Hu (Shanghai Key Laboratory for Reproductive Medicine), Dr Sunhong Chen and Mr Yiping Jin (Department of Histology and Embryology, School of Medicine, Shanghai Jiao Tong University) for their technical assistance. The authors also thank Prof. Peter Reinach for his editorial assistance. This research project was supported by grants from the Science and Technology Commission of Shanghai Municipality

(no.09ZR1416300), Shanghai Leading Academic Discipline Project (no. S30201) and Shanghai Basic Research Project (no.09DJ1400400).

\section{Authors' contributions}

$\mathrm{XY}$ and $\mathrm{YL}$ participated in all aspects of the experiment. QG carried out the immunofluorescent staining. $F Q, W G$ and $Y Z$ partially participated in the Western blots, antibodies generation and sperm-oocyte fusion analyses. ZD conceived of the study, and participated in its design and edited the manuscript. All authors read and approved the final manuscript.

\section{Competing interests}

The authors declare that they have no competing interests.

Received: 10 December 2009

Accepted: 4 February 2010 Published: 4 February 2010

\section{References}

1. Kaji K, Kudo A: The mechanism of sperm-oocyte fusion in mammals. Reproduction 2004, 127(4):423-429.

2. Cho C, Bunch DO, Faure JE, Goulding EH, Eddy EM, Primakoff P, Myles DG: Fertilization defects in sperm from mice lacking fertilin beta. Science 1998, 281:1857-1859.

3. Nishimura H, Cho C, Branciforte DR, Myles DG, Primakoff P: Analysis of loss of adhesive function in sperm lacking cyritestin or fertilin beta. Dev Biol 2001, 233:204-213.

4. Shamsadin R, Adham IM, Nayernia K, Heinlein UA, Oberwinkler H, Engel W: Male mice deficient for germ-cell cyritestin are infertile. Biol Reprod 1999, 61:1445-1451.

5. Busso D, Goldweic NM, Hayashi M, Kasahara M, Cuasnicú PS: Evidence for the involvement of testicular protein CRISP2 in mouse sperm-egg fusion. Biol Reprod 2007, 76:701-708.

6. Ellerman DA, Da Ros VG, Cohen DJ, Busso D, Morgenfeld MM, Cuasnicú PS: Expression and structure-function analysis of de, a sperm cysteine-rich secretory protein that mediates gamete fusion. Biol Reprod 2002, 67:1225-1231. 
7. Inoue N, Ikawa M, Isotani A, Okabe M: The immunoglobulin superfamily protein Izumo is required for sperm to fuse with eggs. Nature 2005, 434(7030):152-153.

8. Sitia R, Braakman I: Quality control in the endoplasmic reticulum protein factory. Nature 2003, 426:891-894

9. Ellerman DA, Myles DG, Primakoff P: A role for sperm surface protein disulfide isomerase activity in gamete fusion: evidence for the participation of ERp57. Dev Cell 2006, 10:831-837.

10. Zhang J, Wu J, Huo R, Mao Y, Lu Y, Guo X, Liu J, Zhou Z, Huang X, Sha J: ERp57 is a potential biomarker for human fertilization capability. Mol Hum Reprod 2007, 13:633-639.

11. Guo W, Qu F, Xia L, Guo Q, Ying X, Ding Z: Identification and characterization of ERp29 in rat spermatozoa during epididymal transit. Reproduction 2007, 133:575-584

12. Magnuson B, Rainey EK, Benjamin T, Baryshev M, Mkrtchian S, Tsai B: ERp29 triggers a conformational change in polyomavirus to stimulate membrane binding. Mol Cell 2005, 20:289-300.

13. Qu F, Ying X, Guo W, Guo Q, Chen G, Liu Y, Ding Z: The role of Zn-alpha2 glycoprotein in sperm motility is mediated by changes in cyclic AMP. Reproduction 2007, 134:569-576.

14. Larson $\mathrm{L}$, Miller DJ: Simple histochemical stain for acrosomes on sperm from several species. Mol Reprod Dev 1999, 52:445-449.

15. Demmer J, Zhou C, Hubbard MJ: Molecular cloning of ERp29, a novel and widely expressed resident of the endoplasmic reticulum. FEBS Lett 1997, 402:145-150

16. Mkrtchian S, Fang C, Hellman U, Ingelman-Sundberg M: A stress-inducible rat liver endoplasmic reticulum protein, ERp29. Eur J Biochem 1998, 251:304-313.

17. Hubbard MJ, McHugh NJ: Human ERp29: isolation, primary structural characterisation and two-dimensional gel mapping. Electrophoresis 2000 21:3785-3796.

18. Kwon OY: Overexpression of ERp29 in the thyrocytes of FRTL-5 cells. Mol Biol Rep 2005, 32:7-13.

19. Shnyder SD, Hubbard MJ: ERp29 is a ubiquitous resident of the endoplasmic reticulum with a distinct role in secretory protein production. J Histochem Cytochem 2002, 50:557-566.

20. MacLeod JC, Sayer RJ, LuCocq JM, Hubbard MJ: ERp29, a general endoplasmic reticulum marker, is highly expressed throughout the brain. J Comp Neurol 2004, 477:29-42.

21. Hermann VM, Cutfield JF, Hubbard MJ: Biophysical characterization of ERp29. Evidence for a key structural role of cysteine 125. J Biol Chem 2005, 280(14):13529-13537.

22. Rainey-Barger EK, Magnuson B, Tsai B: A chaperone-activated nonenveloped virus perforates the physiologically relevant endoplasmic reticulum membrane. J Virol 2007, 81:12996-13004.

23. Yano R, Matsuyama $T$, Kaneko $T$, Kurio $H$, Murayama E, Toshimori $K$, lida H: Bactericidal/Permeability-Increasing Protein (BPI) Is Associated with the Acrosome Region of Rodent Epididymal Spermatozoa. J Androl 2009.

24. Turano C, Coppari S, Altieri F, Ferraro A: Proteins of the PDI family: unpredicted non-ER locations and functions. J Cell Physiol 2002, 193:154-163.

25. Hubbard MJ, McHugh NJ, Carne DL: Isolation of ERp29, a novel endoplasmic reticulum protein, from rat enamel cells evidence for a unique role in secretory-protein synthesis. Eur J Biochem 2000, 267:1945-1957.

26. Liepinsh E, Baryshev M, Sharipo A, Ingelman-Sundberg M, Otting G, Mkrtchian S: Thioredoxin fold as homodimerization module in the putative chaperone ERp29: NMR structures of the domains and experimental model of the $51 \mathrm{kDa}$ dimer. Structure 2001, 9:457-471.

27. Sargsyan E, Baryshev M, Backlund M, Sharipo A, Mkrtchian S: Genomic organization and promoter characterization of the gene encoding a putative endoplasmic reticulum chaperone, ERp29. Gene 2002, 285:127-139.

28. Sargsyan E, Baryshev M, Szekely L, Sharipo A, Mkrtchian S: Identification of ERp29, an endoplasmic reticulum lumenal protein, as a new member of the thyroglobulin folding complex. J Biol Chem 2002, 277:17009-17015.

29. Mkrtchian S, Sandalova T: ERp29, an unusual redox-inactive member of the thioredoxin family. Antioxid Redox Sign 2006, 8:325-337.

30. Gilbert J, Ou W, Silver J, Benjamin T: Downregulation of protein disulfide isomerase inhibits infection by the mouse polyomavirus. J Virol 2006, 80(21):10868-10870.
31. Rainey-Barger EK, Magnuson B, Tsai B: Dimerization of ERp29, a PDI-like protein, is essential for its diverse functions. Mol Biol Cell 2007, 18:1253-1260.

32. Rainey-Barger EK, Magnuson B, Tsai B: The C-terminal domain of ERp29 mediates polyomavirus binding, unfolding and infection. J Virol 2009, 83(3):1483-1491

doi:10.1186/1477-7827-8-10

Cite this article as: Ying et al:: Endoplasmic reticulum protein 29 (ERp29), a protein related to sperm maturation is involved in spermoocyte fusion in mouse. Reproductive Biology and Endocrinology 2010 8:10.

\section{Submit your next manuscript to BioMed Central and take full advantage of:}

- Convenient online submission

- Thorough peer review

- No space constraints or color figure charges

- Immediate publication on acceptance

- Inclusion in PubMed, CAS, Scopus and Google Scholar

- Research which is freely available for redistribution
C Biomed Central 